\title{
Production of geranylgeraniol on overexpression of a prenyl diphosphate synthase fusion gene in Saccharomyces cerevisiae.
}

\section{AUTHOR(S):}

Ohto, Chikara; Muramatsu, Masayoshi; Obata, Shusei; Sakuradani, Eiji; Shimizu, Sakayu

\section{CITATION:}

Ohto, Chikara ... [et al]. Production of geranylgeraniol on overexpression of a prenyl diphosphate synthase fusion gene in Saccharomyces cerevisiae.. Applied microbiology and biotechnology 2010, 87(4): 1327-1334

\section{ISSUE DATE:}

2010-07

URL:

http://hdl.handle.net/2433/128882

\section{RIGHT:}

The original publication is available at www.springerlink.com; この論文 は出版社版でありません。引用の際には出版社版をご確認ご利用くだ さい。; This is not the published version. Please cite only the published version. 
1 Production of geranylgeraniol on overexpression of a prenyl diphosphate synthase

2 fusion gene in Saccharomyces cerevisiae

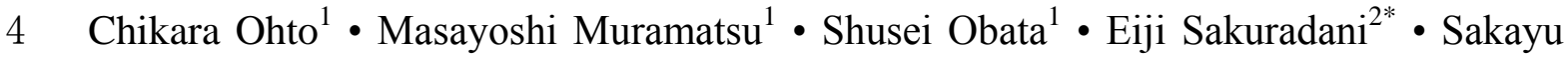

$5 \quad$ Shimizu $^{3}$

6

$7 \quad{ }^{1}$ Bio Research Lab., Toyota Motor Corporation, 1 Toyota-cho, Toyota 471-8572, Japan

$8{ }^{2}$ Division of Applied Life Sciences, Graduate School of Agriculture, Kyoto University,

9 Kitashirakawa-oiwakecho, Sakyo-ku, Kyoto 606-8502. Japan

$10{ }^{3}$ Department of Bioscience and Biotechnology, Faculty of Bioenvironmental Science,

11 Kyoto Gakuen University, Sogabe-cho, Kameoka, Kyoto 621-8555, Japan

13 Correspondence to: * Eiji Sakuradani, Ph.D.

Division of Applied Life Sciences,

Graduate School of Agriculture, Kyoto University,

Kitashirakawa-oiwakecho, Sakyo-ku, Kyoto 606-8502, Japan

Phone: +81-75-753-6114

e-mail: esakura@kais.kyoto-u.ac.jp

Running title: Geranylgeraniol Production by Recombinant Yeast without Sterols 
1 Abstract: An acyclic diterpene alcohol, $(E, E, E)$-geranylgeraniol $(\mathrm{GGOH})$, is one of

2 the important compounds used as perfume and pharmacological agents. A deficiency of

3 squalene (SQ) synthase activity allows yeasts to accumulate an acyclic sesquiterpene

4 alcohol, $(E, E)$-farnesol, in their cells. Since sterols are essential for the growth of yeasts,

5 a deficiency of SQ synthase activity makes the addition of supplemental sterols to the

6 culture media necessary. To develop a GGOH production method not requiring any

7 supplemental sterols, we overexpressed HMG1 encoding hydroxymethylglutaryl-CoA

8 reductase and the genes of two prenyl diphosphate synthases, ERG20 and BTS1, in

9 Saccharomyces cerevisiae. A prototrophic diploid coexpressing HMG1 and the

10 ERG20-BTS1 fusion accumulated GGOH with neither disruption of the SQ synthase

11 gene nor the addition of any supplemental sterols. The GGOH content on the diploid

12 cultivation in a 5 l-jar fermenter reached $138.8 \mathrm{mg} / 1$ under the optimal conditions.

14 KEY WORDS: geranylgeraniol; mevalonate pathway; hydroxymethylglutaryl-CoA reductase; prenyl diphosphate synthase; yeast recombinant 


\section{Introduction}

2

3 An acyclic diterpene alcohol, (E,E,E)-geranylgeraniol $(\mathrm{GGOH})$, is known to be a

4 fragrant constituent of essential oils. It has been used not only as one of the important

5 ingredients in perfumes, but also as a valuable material for the chemical synthesis of

6 pharmacological agents and hydrophobic vitamins such as vitamin A and E (Benford et

7 al. 1999; Hyatt et al. 2002). The diphosphate derivative of GGOH,

$8(E, E, E)$-geranylgeranyl diphosphate (GGPP), is a significant intermediate in the early

9 stage of carotenoid biosynthesis, particularly in plant cells. Although there are eight

10 geometric isomers of GGPP as to the combination of the $E / Z$-conformation at three

11 carbon-carbon double bonds, only the $(E, E, E)$-isomer exhibits significant biological

12 activity (Ogura and Koyama 1998). The chemical synthesis of GGOH has been difficult

13 because of the introduction of three $E$-double bonds into the isoprenoid structure.

14 Although, in the last few years, the chemical synthesis for (all-E)-prenyl alcohols has

15 been performed with relatively high yields (Negishi et al. 2002; Yu et al. 2005),

16 metabolic engineering still exhibits greater potential as to an efficient production of

17 (all-E)-prenyl alcohols, because living cells accomplish a variety of simultaneous 
1 reactions with specific stereo-specificities. We previously reported that a recombinant

2 Saccharomyces cerevisiae overexpressing the genes encoding the enzymes in the

3 mevalonate pathway could produce $145.7 \mathrm{mg} / \mathrm{l}$ of $(E, E)$-farnesol $(\mathrm{FOH})$, suggesting the

4 yeast has phosphatase activity that converts prenyl diphosphates into prenyl alcohols

5 (Ohto et al. 2009b). But it was unclear whether GGPP could be similarly

6 dephosphorylated and secreted into the extracellular fraction to be easily extracted with

7 organic solvent without cell disruption.

8 In all organisms, GGPP is synthesized through an enzymatic condensation reaction

9 of allylic diphosphates such as dimethylallyl diphosphate, $(E)$-geranyl diphosphate and

10 (E,E)-farnesyl diphosphate (FPP) with isopentenyl diphosphate(s). Since two FPPs are

11 also head-to-head condensed to produce squalene (SQ), which is an intermediate during

12 sterol biosynthesis, GGPP biosynthesis would compete with SQ biosynthesis for FPP

13 (Fig. 1). SQ accumulates intracellularly due to overexpression of a Fig. 1

14 3-hydroxy-3-methylglutaryl-CoA reductase gene (HMG1), this enzyme being one of the

15 rate-limiting enzymes in the mevalonate pathway (Donald et al. 1997). Chambon et al.

16 reported that a sterol-auxotrophic yeast deficient in the SQ synthase gene could produce

17 0.74-1.3 mg/l of FOH in a culture (Chambon et al. 1990; Chambon et al. 1991). The 
1 addition of an inhibitor of SQ synthase allowed yeasts to produce FPP and FOH, but not

2 a sufficient amount of GGOH (Bergstrom et al. 1993; Muramatsu et al. 2008b).

3 Recently, with both SQ synthase and phytoene synthase inhibitors, a

4 carotenoid-producing yeast Rhodotorula rubra IFO 0870 could produce $53.7 \mathrm{mg} / \mathrm{l}$ of

5 GGOH (Muramatsu et al. 2008b). The overexpression of an isopentenyl diphosphate

6 delta-isomerase gene and a mutated FPP synthase one in Escherichia coli led to the

7 accumulation of $0.13 \mathrm{mg} / \mathrm{l} \mathrm{GGOH}$ in the cells (Ohto et al. 2009a). In the present study, a

8 recombinant yeast overexpressing the genes encoding enzymes involved in the

9 mevalonate pathway led to the production of $\mathrm{GGOH}$ without the addition of any

10 supplemental sterols. In particular, the coexpression of $H M G 1$ and a prenyl diphosphate

11 synthase fusion gene was effective for the production of GGOH by S. cerevisiae.

Strains 
1 S. cerevisiae strains A451 (ATCC 200589) was purchased from the ATCC (American

2 Type Culture Collection, Manassas, VA). AURGG101, which comprises A451 bearing

3 AUR1-C, was previously described (Ohto et al. 2009b). YPH499 (ATCC 76625) and

4 YPH500 (ATCC 76626) were purchased from Stratagene (La Jolla, CA).

5

6 Construction of YEp expression vectors

7

8 YEp (multicopy-type) expression vectors were constructed from pRS vectors

9 (Stratagene) and YES2 (Invitrogen, Carlsbad, CA), as previously reported (Ohto et al.

10 2009b). The nucleotide sequences of these vectors are available under DDBJ accession

11 No. AB304849-AB304876.

13 Preparation of episome DNA constructs for overexpression

15 Using the primer DNA pairs shown in Fig. 2, the farnesyl diphosphate synthase gene

16 (ERG20) and the geranylgeranyl diphosphate synthase gene (BTS1) were amplified by

17 PCR from pT7-ERG20 and pYES-GGPS (Ohto et al. 2009b), respectively, ligated at the 
1 EcoO109I sites to prepare ERG20 and BTS1 fusion genes, and then inserted into

2 pRS435GAP (DDBJ accession No. AB304858) and pRS445GAP (AB304870). The

3 names and nucleotide sequences of the primers are as follows; SacII-BTS1, ${ }^{\text {'TCC CCG }}$

4 CGG ATG GAG GCC AAG ATA GAT ${ }^{3}$; BTSI-XhoI, ${ }^{5}$ CAA CTC GAG TCA CAA

5 TTC GGA TAA GTG ${ }^{3}$; BTSI-109I, ${ }^{5}$ GCA GGG ACC CCA ATT CGG ATA AGT GGT

6 C C $^{\prime} ;$ 109I-BTS1, 5' GTA GGG TCC CTG GAG GCC AAG ATA GAT G ${ }^{3}$; ERG20-109I,

$7 \quad{ }^{5}$ GCA GGG ACC CTT TGC TTC TCT TGT AAA CT ${ }^{3}$; 109I-ERG20, 5' GTA GGG

8 TCC TCA GAA AAA GAA ATT AGG AG ${ }^{3} ;-21,{ }^{5}$ 'TGT AAA ACG ACG GCC AGT ${ }^{3}$;

9 and T7, 5' TAA TAC GAC TCA CTA TAG GG ${ }^{3}$. The names of the resultant episome

10 DNAs harboring the fusion genes are shown in Fig. 2. The episome DNAs for

11 overexpression of other wild type genes were described previously (Ohto et al. 2009b).

13 Culture conditions for episome DNA-harboring yeasts

15 The transformants with episome DNAs were selected on agar plates of SD medium

16 (BIO101, Vista, CA) lacking the specified amino acids as to the auxotrophic markers at

$1730^{\circ} \mathrm{C}$. They were cultivated in $2.5 \mathrm{ml}$ aliquots of YM7 or YMO medium in glass tubes 
1 by rotary shaking at $130 \mathrm{rpm}$ at $30^{\circ} \mathrm{C}$. YM7 medium comprises the $\mathrm{YM}$ broth (Becton,

2 Dickinson and Company, Franklin Lakes, NJ) adjusted to pH 7.0 with $\mathrm{NaOH}$, and YMO

3 comprises YM7 including 3.0\% (w/v) soybean oil (Nacalai Tesque, Kyoto, Japan).

5 Construction of a diploid recombinant strain

6

7 DNA fragments of the ADE2, HIS3, LYS1 and URA3 coding regions were prepared by

8 PCR from YPH499 genomic DNA based on the sequence in the S. cerevisiae genome

9 database. The ADE2 and HIS3 fragments were introduced into YPH499-originating

10 recombinants using a medium lacking adenine and histidine, and the LYS1 and URA3

11 fragments were introduced into YPH500 using a medium lacking lysine and uracil. The

12 resultant YPH499 recombinant bearing ADE2 and HIS3, named YPH499-AH, and the

13 YPH500 one bearing LYS1 and URA3, named YPH500-KU, were mated on a minimum

14 SD plate to produce diploid prototrophs.

16 Preparation of DNA, RNA and crude enzyme 
1 The yeast transformants were cultivated in $100 \mathrm{ml}$ aliquots of SD or SG medium and

2 then cultured in $300-\mathrm{ml}$ Erlenmeyer flasks at $26^{\circ} \mathrm{C}$ with reciprocal shaking at 120

3 times/min. The cultivation was finished when $\mathrm{OD}_{600}$ reached around 3-4 (corresponding

4 to 23-53 hours), followed by the preparation of DNA, RNA and a crude enzyme

5 solution. The yeast genomic DNA and total RNA were prepared according to the

6 standard procedures. For the preparation of crude enzyme solutions, cells were

7 harvested by centrifugation, disrupted at $4^{\circ} \mathrm{C}$ with glass beads and then suspended in

8 sterilized water. Each suspension was centrifuged at $12,000 \mathrm{rpm}$ at $4^{\circ} \mathrm{C}$ for $10 \mathrm{~min}$, and

9 the resultant supernatant was used for the enzyme assay. Protein concentrations were

10 determined with Protein Assay (Bio-Rad, Hercules, CA).

Northern blot hybridization

14 Probes for Northern blot hybridization were prepared with a PCR DIG Probe Synthesis

15 Kit (Roche Diagnostics, Mannheim Germany) using the templates and primer pairs

16 listed in Table 1. The templates for pT7ERG20, pYESGGPS, and pYHMG1 were

17 described previously (Ohto et al. 2009b). As a control probe, the coding region of the 
$1 \quad \alpha$-tubulin gene, TUB1, was amplified from YPH499 genomic DNA and prepared as a

2 DIG-labeled probe in the same way. Five mg of yeast total RNA per lane was subjected

3 to electrophoresis on a formaldehyde-denaturated agarose gel, followed by transfer to a

4 Hybond $\mathrm{N}$ nylon membrane (GE Healthcare Ltd, Amersham Place, UK), and

5 cross-linked to it according to a conventional method. Northern blot hybridization was

6 carried out with $100 \mathrm{ng} / \mathrm{ml}$ probe DNA at $50^{\circ} \mathrm{C}$ for 24 hours according to the standard

7 protocol for a DIG Easy Hyb Kit (Roche Diagnostics). The signals were detected as

8 chemiluminescence using a DIG Luminescent Detection Kit (Roche), followed by

9 exposure of the blot to X-ray film.

11 Prenyl alcohol production in a jar fermenter

13 The yeast transformants were inoculated from slants into $200 \mathrm{ml}$ aliquots of DOB-based

14 selection medium (BIO101) in 500-ml Erlenmeyer flasks with baffle plates, and cultured at $30^{\circ} \mathrm{C}$ for 30 hours with shaking at $120 \mathrm{rpm}$. Ten $\mathrm{ml}$ of the resultant culture

16 was inoculated into 51 of medium comprising $300 \mathrm{~g}$ of glucose, $100 \mathrm{~g}$ of yeast extract,

$17100 \mathrm{~g}$ of bactopeptone, $550 \mathrm{~g}$ of soybean oil, and $5 \mathrm{ml}$ of Adekanol LG109 (Adeka, 
1 Tokyo Japan). The operational conditions were as previously given (Ohto et al. 2009b).

2 Two milliliters of the culture broth was periodically taken, and the concentrations of

3 glucose, ethanol and acetate were determined as previously reported (Muramatsu et al.

4 2008b). Cell density was measured by directly counting cells in diluted broth on a

5 hematoplate under a phase-constant microscope.

6

$7 \quad$ Extraction of prenyl alcohols and GC/MS analysis

$92.5 \mathrm{ml}$ of methanol was added to $2.5 \mathrm{ml}$ of a main culture, followed by thorough mixing.

10 Five $\mathrm{ml}$ of pentane was added to the mixture, followed by vigorous agitation. After

11 standing for a few minutes, the pentane layer was transferred to a new glass tube for analysis by GC/MS (Ohto et al. 2009b).

\section{Results}

17 Prenyl alcohol production by the recombinants overexpressing the genes of the enzymes 
1 in the mevalonate and prenyl diphosphate pathways under the control of the TDH3

2 promoter

3

4 In order to determine the effects of genes related with the mevalonate and prenyl

5 diphosphate biosynthesis for GGOH production, each gene was introduced individually

6 into S. cerevisiae YPH499 by means of pRS434GAP (AB304854) and pRS435GAP

7 (AB304858), which were YEp vectors bearing the $T D H 3$ promoter that is constitutively

8 active. Although pRS434GAP was used for the expression of HMG1 and pRS435GAP

9 for other genes, these vectors have almost the same sequences except for the selection

10 marker (TRP1 and $L E U 2$, respectively). Pronounced FOH production was observed on

11 the overexpression of $H M G 1$ among the nine genes responsible for prenyl alcohol

12 production, whereas GGOH increased in amount on overexpression of BTS1, HMG1

13 and ERG20, in that order (Fig. 3). The YEp vector elevated the productivity of GGOH

14 5-fold compared with the YIp vector (data not shown). 
1 The PCR fragments of ERG20, BTS1, the BTS1-ERG20 fusion gene and the

2 ERG20-BTS1 fusion gene were inserted into the multiple cloning sites of the YEp vector

3 of pRS435, which is located between the TDH3 promoter and the CYC1 terminator, to

4 prepare pRS435GAP-ERG20, pRS435GAP-BTS1 pRS435GGF and pRS435FGG,

5 respectively, followed by transformation into S. cerevisiae YPH499. As shown in Fig. 2,

6 the two-amino acid linker sequence of Gly-Ser between the two prenyl diphosphate

7 synthases was designed for the fusion of their enzymes. The fusion genes are supposed

8 to lead the formation of a fused protein comprised of the BTS1 and the ERG20.

9 Although the host strain and the recombinant pRS435GAP-ERG20/YPH499 produced

10 little GGOH in the culture broth, the overexpression of BTS1 was an effective means of

11 enhancing GGOH production. This effect was remarkably observed on fusion with the

$12 E R G 20$ gene synergistically, being particularly higher, eight-fold, on fusion with ERG20

13 at 6 bp downstream from BTS1 (pRS435GGF) compared with BTS1 alone

14 (pRS435GAP-BTS1 in Fig. 4).

We further introduced this effective episome of pRS435GGF for GGOH production into SQ-overproducing strain pRS435GAP-HMG1/YPH499 (Ohto et al. 2009b). The 
1 hybridization, where the probe hybridizing to the $\alpha$-tubulin gene, $T U B 1$, was used as a

2 control (Fig. 5). Using the probes of ERG2O and BTS1, strong signals of $3.1 \mathrm{~kb}$ were

3 detected for pRS435GGF/YPH499 and pRS435GGF/pRS434GAP-HMG1/YPH499

4 harboring the BTS1-ERG20 fusion gene. HMG1 was also confirmed to be

5 overexpressed in the recombinants having pRS434GAP-HMG1. With YPH499 as the

6 host strain, considerably strong ERG20 expression was observed but a BTS1 transcript

7 was not detected. The recombinant overexpressing three effective components (BTS1,

8 ERG20, and $H M G 1$ ) was used as a promising producer for GGOH production in a jar

9 fermenter.

11 Jar fermenter production

13 Prototrophic cells generally exhibit tolerance to environmental stresses such as osmotic

14 pressure, alcohol and other chemical compounds.

15 pRS435GGF/pRS434GAP-HMG1/YPH499 was transformed with supplementary genes

16 (ADE2 and HIS3) and then mated with YPH500-KU bearing LYS1 and URA3 to

17 generate a prototroph diploid of pRS435GGF/pYESHMG044/YPH501-AHKU, 
1 designated as the GGOH strain. The important factors (cell growth rate in the

2 logarithmic phase and cell density in the stationary phase) for the commercial

3 production of $\mathrm{GGOH}$ improved with the disappearance of each nutrient requirement

4 marker (data not shown). As the GGOH strain showed the best GGOH production (8.3

$5 \mathrm{mg} / \mathrm{l}$ ) in a test tube containing YMO medium, it was cultivated in a 5 l-jar fermenter

6 with sufficient aeration and glucose as a carbon source for GGOH production (Fig. 6).

7 After prolonged fermentation for $133.5 \mathrm{~h}$, the GGOH and SQ production reached 138.8

$8 \mathrm{mg} / \mathrm{l}$ and $60.0 \mathrm{mg} / \mathrm{l}$, respectively. Other prenyl alcohol production was negligible during

9 the whole fermentation. The $\mathrm{FOH}$ production reached a maximal level of $2.09 \mathrm{mg} / \mathrm{l}$ at

$10 \quad 61.5 \mathrm{~h}$, and gradually decreased thereafter.

\section{Discussion}

15 Having three carbon atoms yielding sixteen geometrical isomers, time-consuming

16 purification steps are inevitable for the chemical synthesis of GGOH. In contrast, for

17 short-chain prenyl diphosphates having not more than 20 carbon atoms, organisms are 
1 able to synthesize all-E isomers through type I prenyl transferases (Ogura and Koyama

2 1998). Hence, we anticipated that a fermentation process comprising the synthesis of

3 all-E prenyl diphosphate isomers would be highly advantageous for chemical synthesis,

4 especially for that of geranylgeranyl diphosphate, which has four carbon-carbon double

5 bonds. Although the metabolic pathway for GGPP has been revealed in various

6 organisms (Fig. 1), it has so far been difficult to establish overproduction technology

7 based on biochemical pathways (Barkovich and Liao 2001; Ohto et al. 2009a). The low

8 activity of GGPP synthase in $S$. cerevisiae is a crucial problem for GGOH production,

9 because GGPP is supposed to be utilized only as an isoprenoid moiety of

10 geranylgeranylated proteins, and FPP, one of the substrates for GGPP synthase, is

11 converted into various compounds, mainly into SQ (Fig. 1).

Co-overexpression of BTS1 in the YIp vector and HMG1 in the YEp vector for

13 GGOH overproduction was ineffective compared to the overexpression of HMGl in the

14 YEp vector in S. cerevisiae A451 for FOH overproduction, as previously reported (Ohto et al. 2009b). Then, we introduced all mevalonate pathway and the prenyl diphosphate

16 pathway-related genes into YEp expression vectors that are maintained as multicopy

17 vectors. Overexpression of BTS1 and HMG1 resulted in more effective production of 
$1 \mathrm{GGOH}$, and the YEp vector proved to be superior to the YIp vector for GGOH

2 production (Fig. 3). It was interesting that the overexpression of ERG20 would be the

3 third significant factor for GGOH production, regardless of the high level expression in

4 wild type cells. The enzyme encoded by BTS1 catalyzes the condensation of FPP and

5 isopentenyl diphosphate into GGPP, but is incapable of utilizing C5-allylic diphosphate

6 substrates (Jiang et al. 1995). A supply of FPP depending upon the expression of ERG20

7 must be inevitable for GGOH overproduction.

8 It was reported that type I prenyl diphosphate syntheses retain sufficient activity as

9 fusion proteins with maltose binding protein and glutathione S-transferase (Ohto et al.

10 1998). Based on this information, we designed fusions of ERG20 and BTS1, and found

11 that the overexpression of the BTS1-ERG20 fusion gene was the most effective among

12 the single or fused genes of prenyl diphosphate synthases we had tested (Fig. 4). Before

13 this study, some natural fused genes had been known for diterpenoid biosynthesis. In the

14 first stage of carotenoid biosynthesis, it was reported that a single gene coded for a

15 protein with both activities of prephytoene diphosphate synthase and phytoene synthase

16 (Velayos et al. 2000). The formation of a gibberellic acid skeleton in fungi is achieved

17 through a bifunctional diterpene cyclase comprising two domains, ent-copalyl 
1 diphosphate synthase responsible for cyclization of GGPP and ent-kaurene synthase

2 (Kawaide 2006). Moreover, there has been an attempt to produce capsidiol using an

3 artificial fusion gene of FPP synthase and sesquiterpene cyclase. The amount of

4 epi-aristolochene produced by the fused enzyme was considerably higher than that by a

5 mixture of the two wild type enzymes (Brodelius 2002). The present data also show that

6 the fused enzyme, which is involved in more than two successive enzymatic reactions in

7 a metabolic pathway, enhances the production of hydrophobic compounds in an aqueous

8 environment. The adjacency of two active sites would be convenient for preventing the

9 first product from diffusing into the aqueous solution as small particles before the next

10 enzymatic reaction, and the fused protein might be more stable in the recombinant

11 strain.

Because of the ability of soybean oil to improve prenyl alcohol productivity

13 (Muramatsu et al. 2008a), we cultivated the diploid prototroph bearing HMGl and the

14 BTS1-ERG20 fusion gene in the optimized medium. A diploid prototroph are commercially attractive for a high growth rate shortening the cultivation period, alcohol

16 tolerant, and broad selectivity as to medium, leading to a low cost. The maximal

17 production of GGOH here $(138.8 \mathrm{mg} / \mathrm{l})$ is a significant value compared with other 
1 terpenoids produced by recombinants, such as limonene $(5 \mathrm{mg} / \mathrm{l})$, taxadiene $(1.3 \mathrm{mg} /)$,

2 and lycopene (22 mg/l) (Carter et al. 2003; Huang et al. 2001; Kim and Keasling 2001).

3 This is the first report on the overproduction of GGOH by a microorganism. It had

4 so far been difficult to overproduce hydrophobic terpenoids including GGOH with

5 metabolically-engineered microorganisms. However, the prospects are bright because

6 the fusion gene of BTS1-ERG20 showed such impressive ability. Further investigation

7 of the on expression of other genes involved in the mevalonate and prenyl diphosphate

8 pathways is necessary, and the difference between multi-copy genome integration and

9 episome retention has to be elucidated for the improvement of GGOH production.

11 Acknowledgement We thank Ms. Chiharu Mori, Ms. Yoshie Tsukahara, Ms. Kazuyo

12 Suzuki and Ms. Kumi Terada for their technical assistance.

\section{References}

16 Barkovich R, Liao JC, (2001) Metabolic engineering of isoprenoids. Metab Eng 3:27-39

17 Benford HL, Frith JC, Auriola S, Mönkkönen J, Rogers MJ, (1999) Farnesol and 
1 geranylgeraniol prevent activation of caspases by aminobisphosphonates:

2 biochemical evidence for two distinct pharmacological classes of bisphosphonate drugs. Mol Pharmacol 56:131-140

4 Bergstrom JD, Kurtz MM, Rew DJ, Amend AM, Karkas JD, Bostedor RG, Bansal VS,

5 Dufresne C, Vanmiddlesworth FL, Hensens OD, Liesch JM, Zink DL, Wilson KE,

6 Onishi J, Milligan JA, Bills G, Kaplan L, Nallin-Omstead M, Jenkins RG, Huang L,

7 Meinz MS, Quinn L, Burg RW, Kong YL, Mochales S, Mojena M, Martin I, Pelaez F, Diez MT, Alberts AW, (1993) Zaragozic acids: a family of fungal metabolites that are picomolar competitive inhibitors of squalene synthase. Proc Natl Acad Sci USA 90:80-84

11 Brodelius M, Lundgren A, Mercke P, Brodelius PE, (2002) Fusion of farnesyl diphosphate synthase and epi-aristolochene synthase, a sesquiterpene cyclase involved in capsidiol biosynthesis in Nicotiana tabacum. Eur $\mathrm{J}$ Biochem 269:3570-3577

15 Carter OA, Peters RJ, Croteau R, (2003) Monoterpene biosynthesis pathway construction in Escherichia coli. Phytochemistry 64:425-433

17 Chambon C, Ladeveze V, Oulmouden A, Servouse M, Karst F, (1990) Isolation and 
1 properties of yeast mutants affected in farnesyl diphosphate synthetase. Curr Genet $18: 41-46$.

3 Chambon C, Ladeveze V, Servouse M, Blanchard L, Javelot C, Vladescu B, Karst F, (1991) Sterol pathway in yeast. Identification and properties of mutant strains defective in mevalonate diphosphate decarboxylase and farnesyl diphosphate synthetase. Lipids 26:633-636

7 Donald KA, Hampton RY, Fritz IB, (1997) Effects of overproduction of the catalytic domain of 3-hydroxy-3-methylglutaryl coenzyme A reductase on squalene synthesis in Saccharomyces cerevisiae. Appl Environ Microbiol 63:3341-3344

Huang Q, Roessner CA, Croteau R, Scott AI, (2001) Engineering Escherichia coli for the synthesis of taxadiene, a key intermediate in the biosynthesis of taxol. Bioorg Med Chem 9:2237-2242

Hyatt JA, Kottas GS, Effler J, (2002) Development of synthetic routes to D,L- $\alpha$ -tocopherol (vitamin E) from biological produced geranylgeraniol. Org Process Res Dev 6:782-787 
1 Kawaide H, (2006) Biochemical and molecular analyses of gibberellin biosynthesis in

2 fungi. Biosci Biotechnol Biochem 70: 583-590

3 Kim SW, Keasling JD, (2001) Metabolic engineering of the nonmevalonate isopentenyl

4 diphosphate synthesis pathway in Escherichia coli enhances lycopene production.

$5 \quad$ Biotechnol Bioeng 72:408-415

6 Muramatsu M, Ohto C, Obata C, Sakuradani E, Shimizu S, (2008a) Various oils and

7 detergents enhance the microbial production of farnesol and related prenyl alcohols.

$8 \quad$ J Biosci Bioeng 106:263-267

9 Muramatsu M, Ohto C, Obata C, Sakuradani E, Shimizu S, (2008b) Accumulation of

10 prenyl alcohols by terpenoid biosynthesis inhibitors in various microorganisms.

11 Appl Microbiol Biotechnol 80:589-595

12 Negishi E, Liou SY, Xu C, Huo S, (2002) A novel, highly selective, and general methodology for the synthesis of 1,5-diene-containing oligoisoprenoids of all

14 possible geometrical combinations exemplified by an iterative and convergent synthesis of coenzyme Q(10). Org Lett 4:261-264

16 Ogura K, Koyama T, (1998) Enzymatic aspects of isoprenoid chain elongation. Chem 
1 Ohto C, Muramatsu M, Obata C, Sakuradani E, Shimizu S, (2009a) Prenyl alcohol

2 production by expression of exogenous isopentenyl diphosphate isomerase and

3 farnesyl diphosphate synthase genes in Escherichia coli. Biosci Biotechnol Biochem 73:186-188

Ohto C, Muramatsu M, Obata C, Sakuradani E, Shimizu S, (2009b) Overexpression of the gene encoding HMG-CoA reductase in Saccharomyces cerevisiae for production of prenyl alcohols. Appl Microbiol Biotechnol 82:837-845

8 Ohto C, Nakane H, Hemmi H, Ohnuma S, Obata S, Nishino T, (1998) Overexpression of an archaeal geranylgeranyl diphosphate synthase in Escherichia coli cells. Biosci Biotechnol Biochem 62:1243-1246

11 Velayos A, Eslava AP, Iturriaga EA, (2000) A bifunctional enzyme with lycopene cyclase and phytoene synthase activities is encoded by the carRP gene of Mucor circinelloides. FEBS J 267:5509-5519

14 Yu JS, Kleckley TS, Wiemer DF, (2005) Synthesis of farnesol isomers via a modified Wittig procedure. Org Lett 7:4803-4806 


\section{$1 \quad$ Figure legends}

2 Fig. 1 Isoprenoids biosynthesis pathway. Black arrows indicate the sequence of

3 enzyme reactions. The corresponding genes were cloned from $S$. cerevisiae and

4 overexpressed in this study. The MEP (methylerythritol phosphate) pathway and the

5 mevalonate pathway are known for isopentenyl diphosphate (C5 isoprenoid) synthesis.

6 The MEP pathway is a prokaryotic pathway in bacteria and some unicellular eukaryotes.

7 The mevalonate pathway is the only route to C5 isoprenoid in animals, plants (except

8 plastids), archaea and fungi including S. cerevisiae. FPP, (E,E)-farnesyl diphosphate;

9 FOH, (E,E)-farnesol; NOH; $(E)$-nerolidol; GGPP, (E,E,E)-geranylgeranyl diphosphate;

$10 \mathrm{GGOH},(E, E, E)$-geranylgeraniol.

12 Fig. 2 Typical constructs of prenyl diphosphate synthase and those fusion genes.

13 ERG20 and BTS1 were fused at the EcoO109I site added by means of PCR with primers

14 (gray triangles), and then inserted into the pRS435GAP expression vector. ERG20,

15 farnesyl diphosphate synthase gene; BTS1, geranylgeranyl diphosphate synthase gene;

16 GS, glycine-serine residues. 
1 Fig. 3 FOH and GGOH production by recombinants overexpressing genes in the

2 mevalonate and prenyl diphosphate pathways. The FOH (A) and GGOH (B) production

3 by the recombinants of the YPH499 strain overexpressing HMG1 and other genes with

4 pRS435GAP. The enzyme genes (ERG10, HMGS, HMG1 ERG12, ERG8, IDI1, ERG20

5 and BTS1) correspond to those in Fig. 1. WILD indicates the YPH499 wild type as a

6 control strain. The recombinants were cultivated in glass tubes containing $2.5 \mathrm{ml}$ of

$7 \quad$ YM7 medium with shaking $(130 \mathrm{rpm})$ at $30^{\circ} \mathrm{C}$ for 7 days.

9 Fig. 4 GGOH production by recombinants. The recombinants were cultivated in glass

10 tubes containing $2.5 \mathrm{ml}$ of $\mathrm{YM} 7$ medium with shaking $(130 \mathrm{rpm})$ at $30^{\circ} \mathrm{C}$ for 7 days.

11 YPH499, host strain; ERG20, pRS435GAP-ERG20/YPH499; BTS1, pRS435GAP-BTS1/YPH499;

FGG,

pRS435FGG/YPH400;

GGF, pRS435GGF/YPH499.

15 Fig. 5 Northern blot hybridization. In each panel the four lanes contain YPH499 wild type (-), pRS435GGF/YPH499 (GGF), pRS434GAP-HMG1/YPH499 (HMG1, -), and 
1 were determined with RNA ladder marker (Nippon Gene, Tokyo, Japan). The

2 recombinants were cultivated in glass tubes containing $2.5 \mathrm{ml}$ of YM7 medium with

3 shaking $(130 \mathrm{rpm})$ at $30^{\circ} \mathrm{C}$ for 3 days.

4

5 Fig. 6 Five 1-jar fermenter cultivation of the GGOH strain.

6 The culture conditions are given under Materials and Methods. 
Table 1 Hybridization probes for Northern blot hybridization

\begin{tabular}{|c|c|c|c|c|}
\hline Probe No. & Target gene & Template & Primer $1^{\mathrm{a}}$ & Primer $2^{b}$ \\
\hline I & ERG20 & pT7ERG20 & SCFPS1 & SCFPS2 \\
\hline II & BTS1 & pYESGGPS & BTS1(1-21) & BTS1(1008-982) \\
\hline III & $H M G 1$ & pYHMG1 & HMG1(1267-1293) & HMG1(2766-2740) \\
\hline \multicolumn{5}{|c|}{${ }^{\mathrm{a}}$ BTS1(1-21), ${ }^{5}$ ATG GAG GCC AAG ATA GAT GAG ${ }^{3}$; HMG1(1267-1293), ${ }^{5}$ AAC } \\
\hline \multicolumn{5}{|c|}{ TTT GGT GCA AAT TGG GTC AAT GAT ${ }^{3}$; SCFPS1, ${ }^{5}$ ATG GCT TCA GAA AAA } \\
\hline \multicolumn{5}{|c|}{ GAA ATT AG ${ }^{3}$} \\
\hline \multicolumn{5}{|c|}{${ }^{\mathrm{b}}$ BTS1(1008-982), 5'TCA CAA TTC GGA TAA GTG GTC ${ }^{3}$; HMG1(2766-2740), } \\
\hline \multicolumn{5}{|c|}{${ }^{5}$ TCC TAA TGC CAA GAA AAC AGC TGT CAC ${ }^{3} ;$ SCFPS $2,{ }^{5}$ CTA TTT GCT TCT } \\
\hline
\end{tabular}




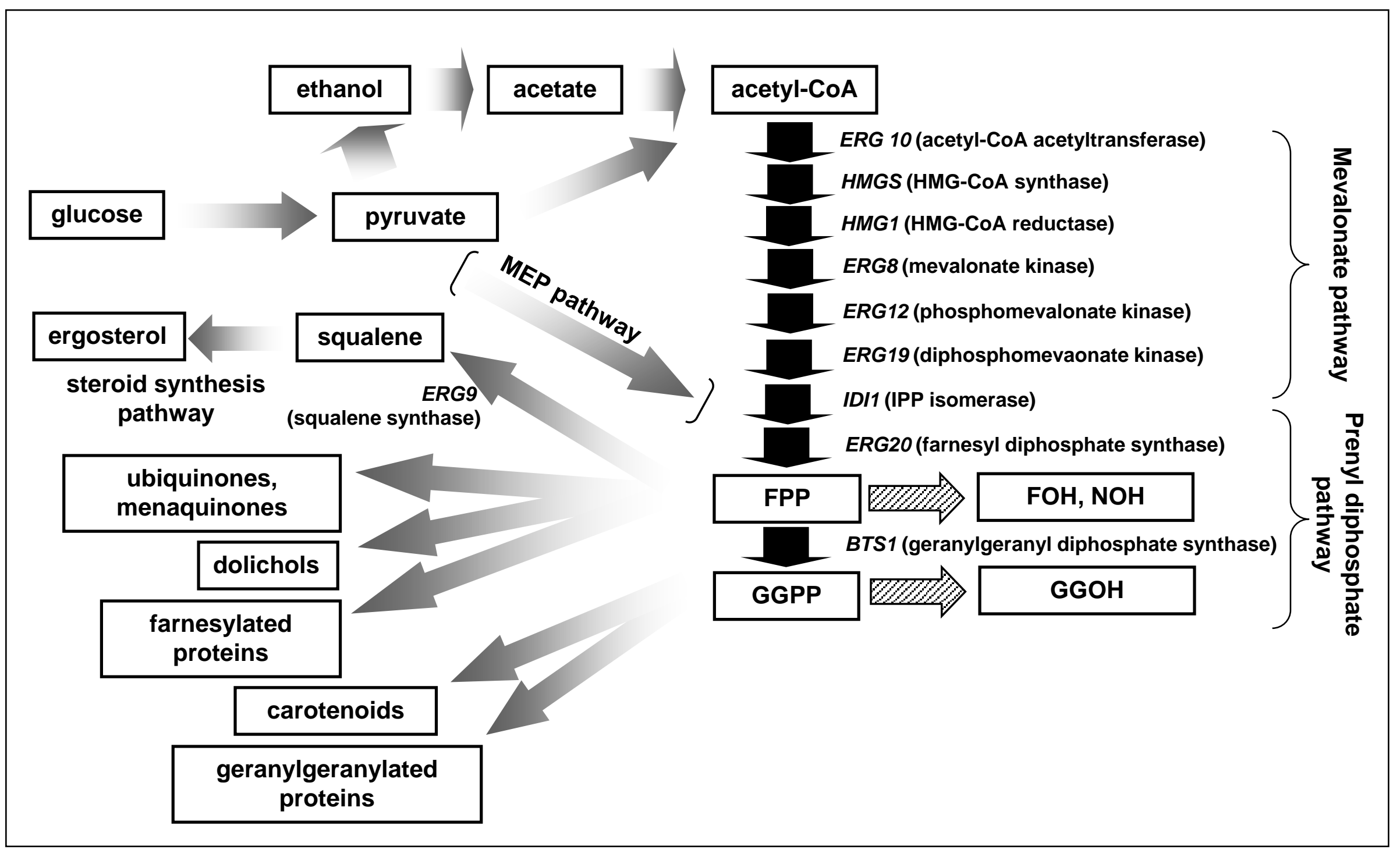

Fig. 1, Ohto et al. 

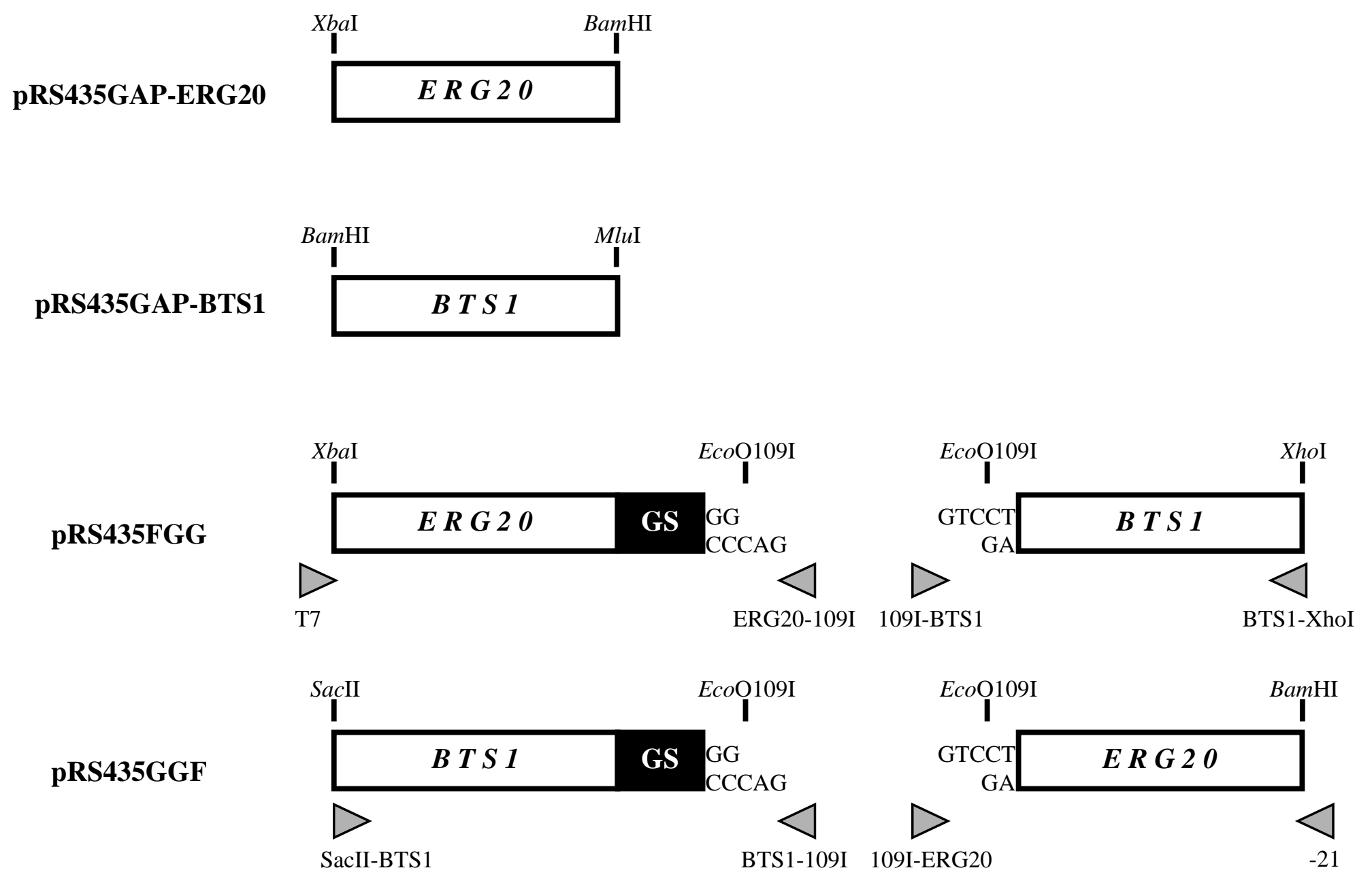

Fig. 2 Ohto et al. 

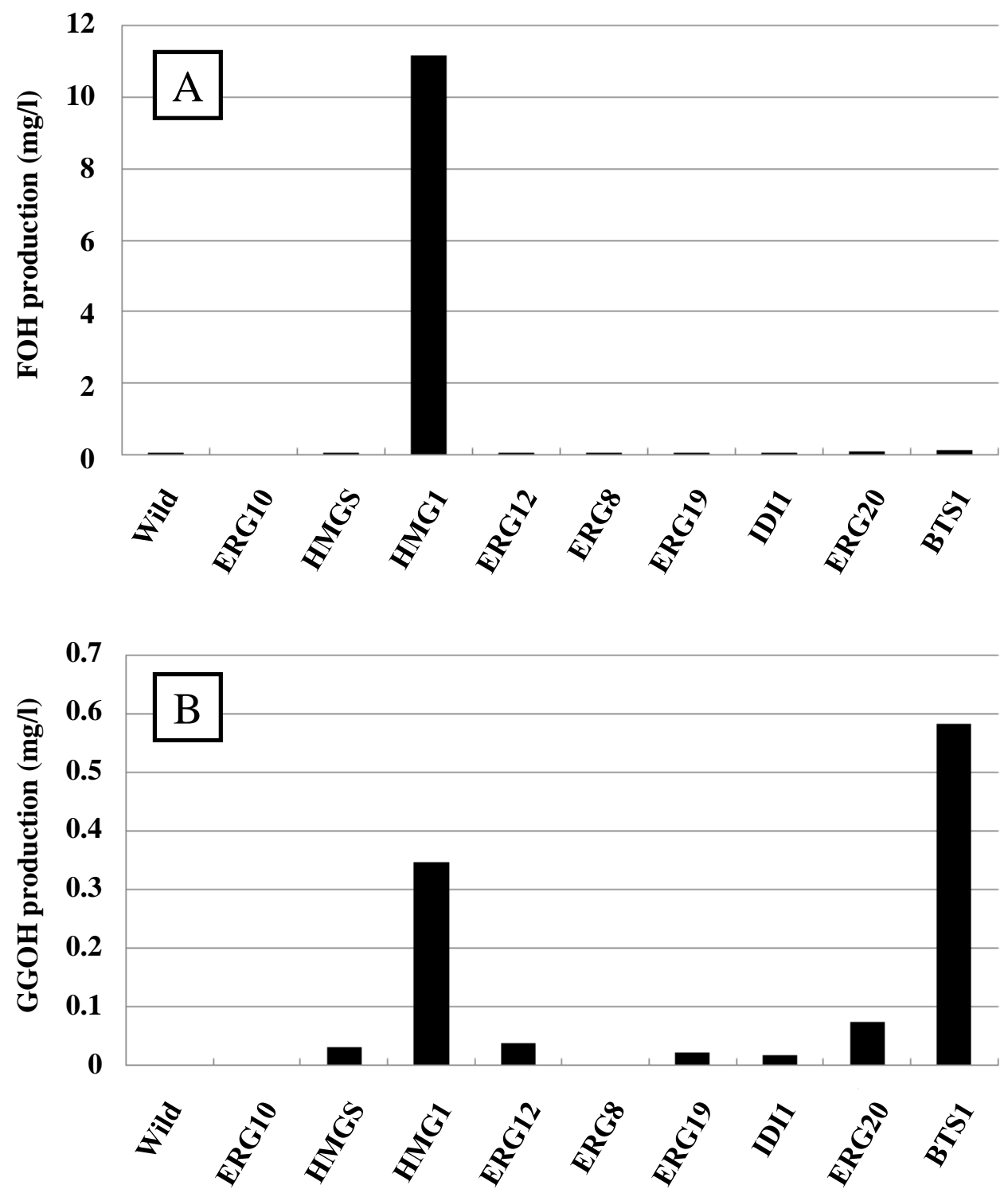

Fig. 3 Ohto et al. 


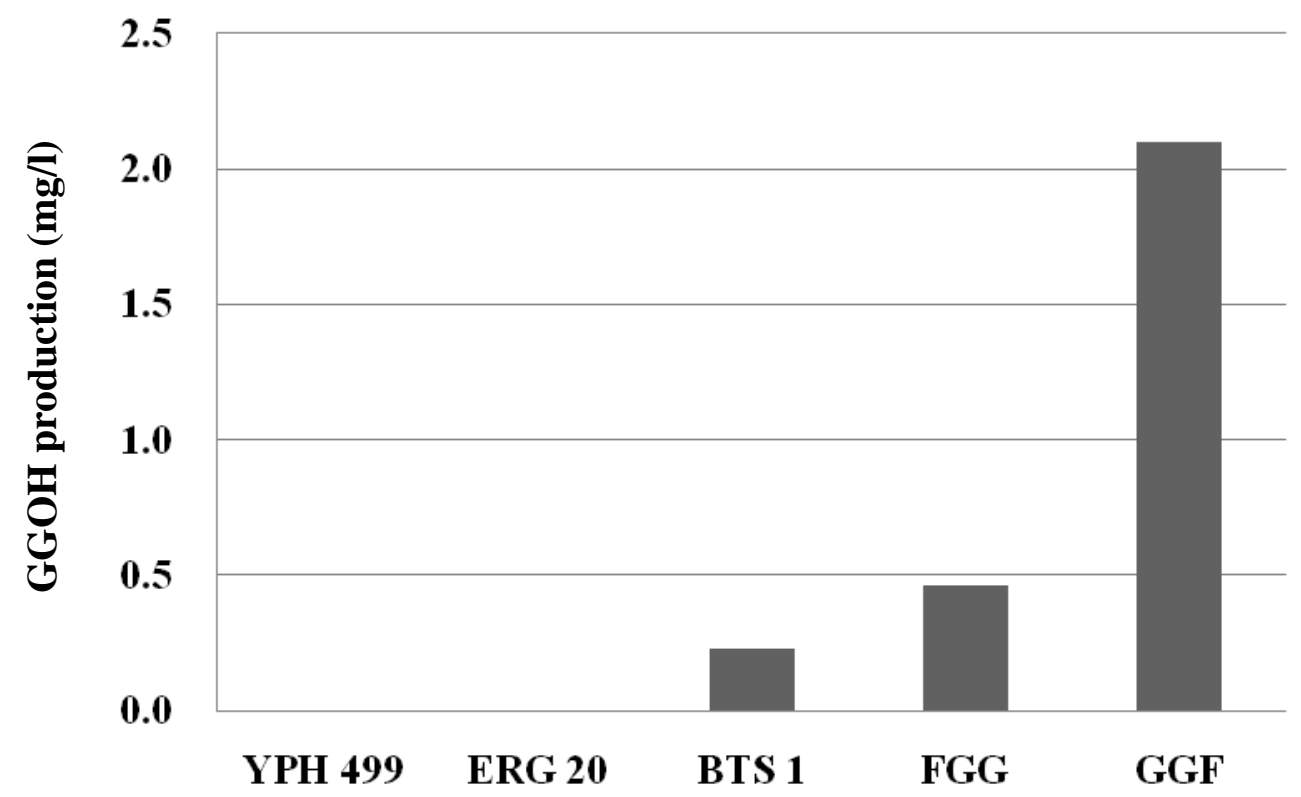

Fig. 4 Ohto et al. 


\section{Y P H 499}

\section{probe:TUB1}

HMG1

岕, 岕

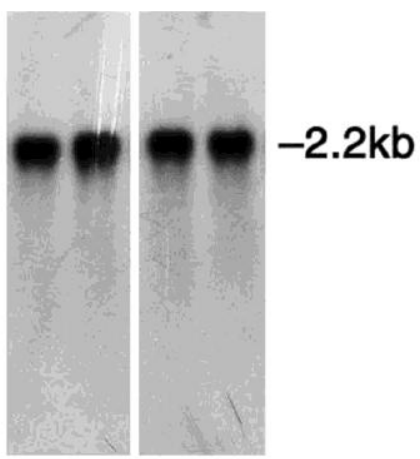

\section{probe:ERG20}

HMG1

, 岕, 荡

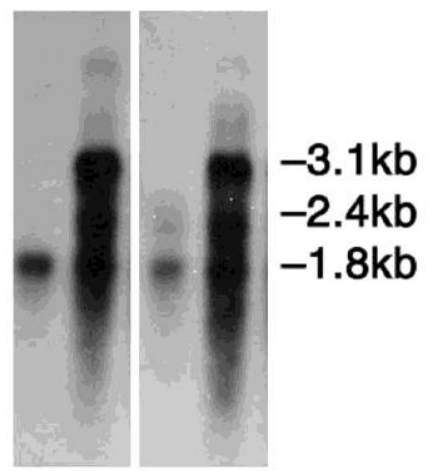

probe:BTS1

HMG1

, 岕, 峛

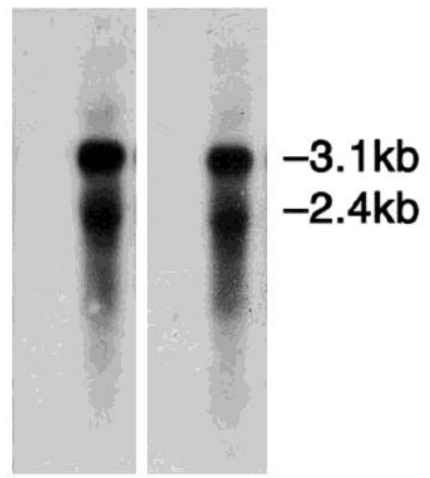

\section{probe:HMG1}

HMG1

, 岕, 岕

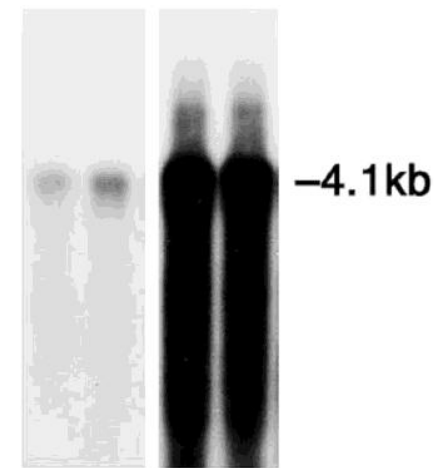

Fig. 5 Ohto et al. 


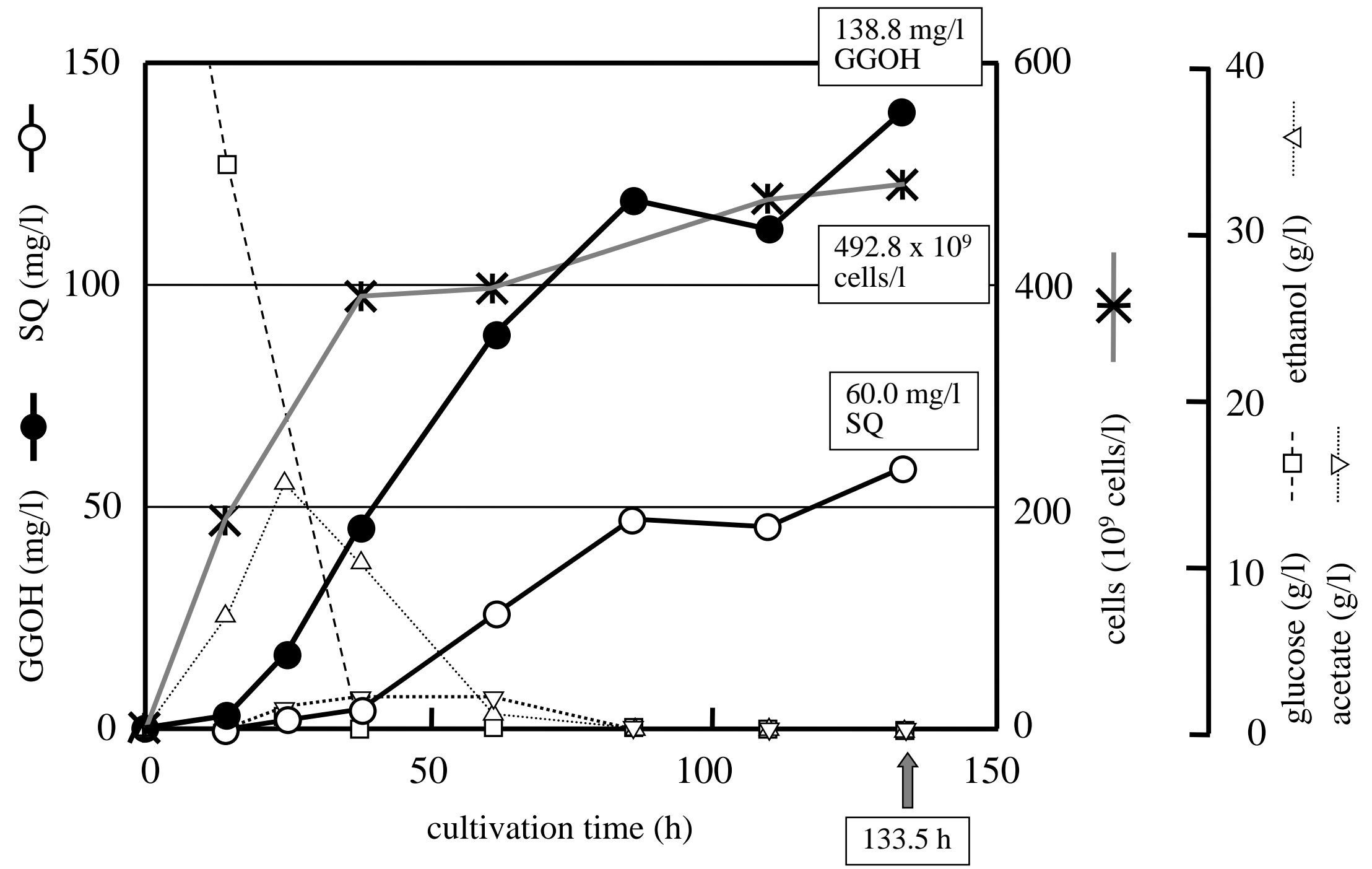

Fig. 6 Ohto et al. 